\title{
A Time Series ANN Approach for Weather Forecasting
}

\author{
Neeraj Kumar ${ }^{1}$, Govind Kumar Jha ${ }^{2}$ \\ ${ }^{1}$ Associate Professor and Head Deptt. Of Computer Science, Nalanda College Of \\ Engineering Chandi(Bihar) \\ ${ }^{2}$ Assistant Professor, Deptt. Of Computer Engineering, GLA University, \\ Mathura(UP), India \\ ${ }^{1}$ javaneraj@gmail.com, ${ }^{2}$ gvnd. jha@gmail.com
}

\begin{abstract}
:
Weather forecasting is most challenging problem around the world. There are various reason because of its experimented values in meteorology, but it is also a typical unbiased time series forecasting problem in scientific research. A lots of methods proposed by various scientists. The motive behind research is to predict more accurate. This paper contribute the same using artificial neural network (ANN) and simulated in MATLAB to predict two important weather parameters i.e. maximum and minimum temperature. The model has been trained using past 60 years of real data collected from(1901-1960) and tested over 40 years to forecast maximum and minimum temperature. The results based on mean square error function (MSE) confirm, this model which is based on multilayer perceptron has the potential to successful application to weather forecasting.
\end{abstract}

\section{Keywords:}

Artificial neural network, Multilayer perceptron, Time series analysis, Mean Square error function, MATLAB.

\section{INTRODUCTION}

Weather forecasting is one of the most important types of forecasting because agriculture sectors as well as many industries are largely dependent on the conditions of weather [1]. The present technology uses numerical model based weather forecasting which involves a large number of calculations made very rapidly using supercomputers.

Mohsen Hayati and Zahra Mohebi worked on one day ahead prediction of weather parameters [14].Suarjit Chattopadhyay ,Department of Mathematics, Techno Model School proposed an Artificial Neural Network based predictive model for predicting average summer-monsoon rainfall [12 ]. Chaw Thet Zan and Thinn Thu Naing of University of Computer Studies, Yangon presented Hidden Markov Models (HMMs) approach for forecasting rainfall time series data for Myanmar rainy season [13]. Paras, Sanjay Mathur, Avinash Kumar, and Mahesh Chandra found that the feature based forecasting model can make predictions with high degree of accuracy [1].

DOI : $10.5121 /$ ijctcm.2013.3102 
There is a scope of improvement in the accuracy of forecasts made by these models when considering the benefits that it has. The idea of incorporating human intelligence together with above discussed model can result in enormous improvement in accuracy of weather forecasting [2]. The objective of this study is to develop a model which comprises this intelligent behavior. The parameters which defining the weather conditions like minimum and maximum temperature ,average rainfall etc., vary continuously with time, forming time series of each parameter which can be used to develop a forecasting model that uses this time series data.

This paper is organized as follows: a brief overview of Artificial Neural Network ,Multilayer perceptron, Back-propagation algorithm, Time Series Analysis and MATLAB are discussed in section II, the data groups and data variables are described in section III, Experimental results obtained using the proposed network and generalisation capacity of model are listed in section $\mathrm{IV}$, and finally we conclude this paper in section V.

\section{NEURAL NETWORK MODEL}

The development of Artificial Neural Network started 50 years ago to mimic the human ability to adapt the changing circumstances and the current environment. It consist of a large number of highly interconnected processing elements called neurons works together to solve specific problems, as shown in Fig1. An artificial neuron is characterized by, architecture (connection between neurons, training or learning determining weights on the connections, activation function [3]. A neural network resembles the human brain in two respects:

1. Knowledge is acquired by the network through a learning process.

2. Inter-neuron connection strengths known as synaptic weights are used to store the knowledge.

The most interesting fact about neural networks is the possibility of learning. A neural network is an adaptive system that changes its structure based on external or internal information that flows through the network during the learning phase [4]. It is used to learn patterns and relationships in data. The learning rules enables a network to gain knowledge from available data and apply that knowledge to train the network [3].

There are three major learning paradigms, each corresponding to a particular abstract learning task. These are supervised learning, unsupervised learning and reinforcement learning. In Supervised Learning, a commonly used function is the mean-squared error, which tries to minimize the average squared error between the network's output, $\mathrm{f}(\mathrm{x})$, and the target value y over all the pairs. In order to minimize this function using gradient descent for the class of neural networks called multilayer perceptrons, we obtain the common and well-known backpropagation algorithm for training neural networks [4]. 


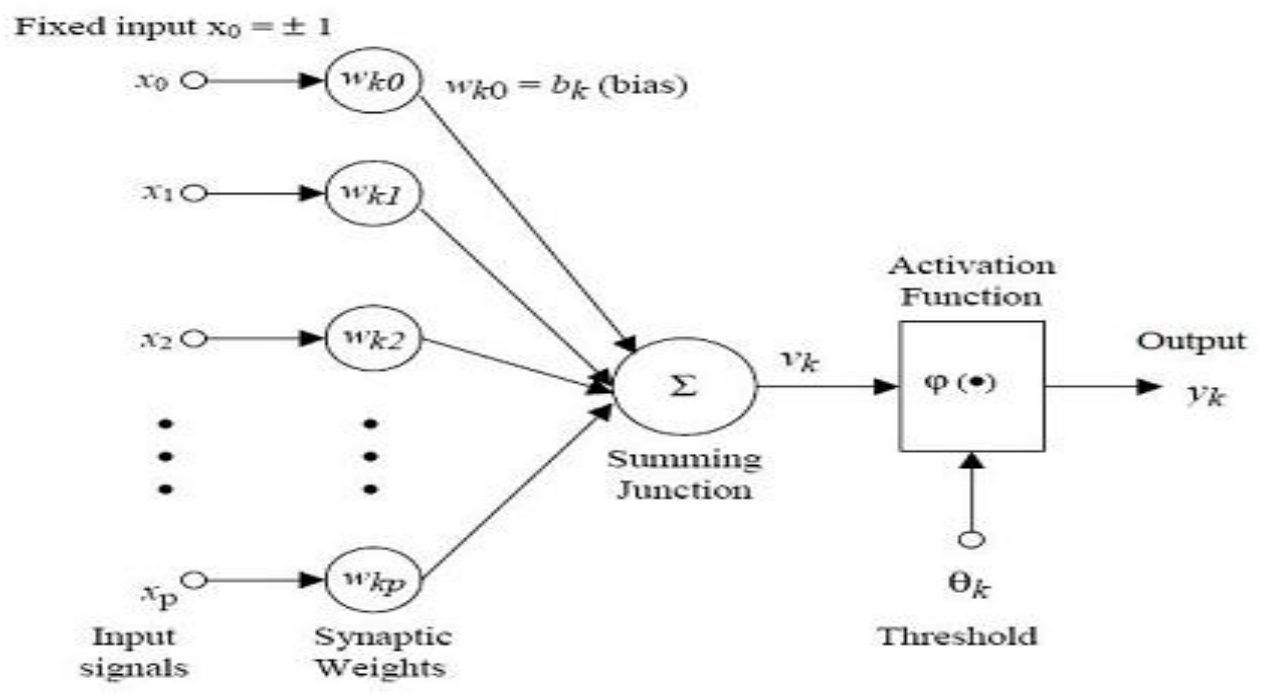

Fig1: Artificial Neural Network.

\section{A.Multi Layer Perceptron Network}

A multilayer perceptron (MLP) is a feed forward artificial neural network model that maps sets of input data onto a set of appropriate output. An MLP consists of multiple layers of nodes in a directed graph, with each layer fully connected to the next layer. It utilizes a supervised learning technique called error- backpropagation for training the network [5][6]. It is a modification of the standard linear perceptron, which can distinguish data that is not linearly separable [7].

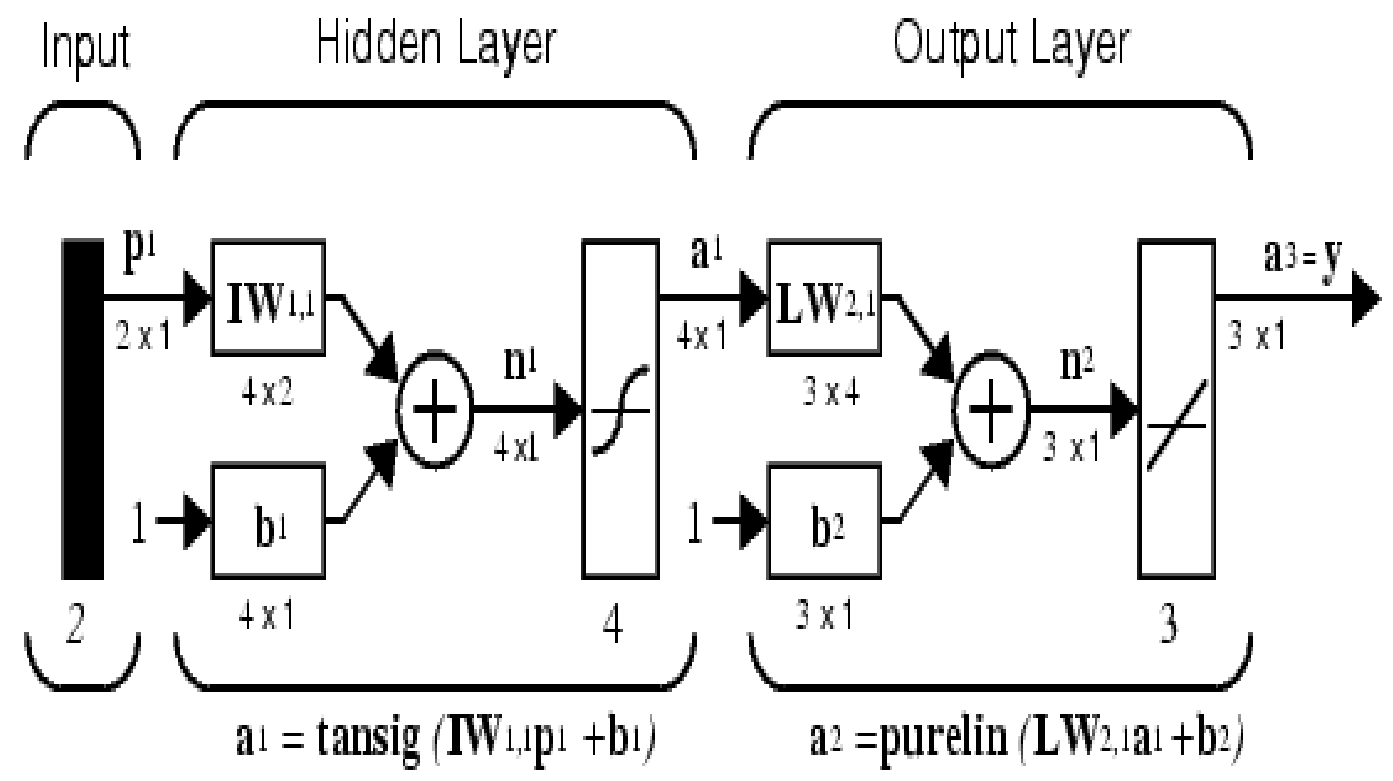

Fig 2: Multilayer Perceptron Network. 
The two main activation functions used in current applications are both sigmoids, and are described by

$$
\phi\left(y_{i}\right)=\tanh \left(v_{i}\right) \text { and } \phi\left(y_{i}\right)=\left(1+e^{-v_{i}}\right)^{-1},
$$

in which the former function is a hyperbolic tangent function that ranges from -1 to 1 , and the latter is equivalent in shape but ranges from 0 to $1 . \mathrm{y}_{\mathrm{i}}$ is the output across the ith neuron and $\mathrm{v}_{\mathrm{i}}$ is the weighted sum of the input synapses[8].

It can implement arbitrary complex input/output mappings or decision surfaces separating different patterns. Multiple-layer networks are quite powerful. For instance, a network of two layers, where the first layer is sigmoid and the second layer is linear, can be trained to approximate any function (with a finite number of discontinuities) arbitrarily well. Such types of network is used extensively in Back-propagation Network. [3]

Back propagation is a systematic method of training for multi layer ANN. It is based upon a strong mathematical foundation when it is not highly practical. It is a multi-layer feed-forward network which used extended gradient descent based delta learning rule, commonly known as Error-Back propagation rule. Because in a gradient descent method minimises the total squared error of the output computed by the network. The network is trained by supervised learning algorithm. The aim of this network is to train the network to achieve a balance between the ability to respond correctly to the input patterns that are used for training and the ability to provide good responses to the input that are similar. This algorithm covers a wide area of applications like, Optical character Recognition, Image Compression, Data Compression, Face Recognition etc.

\section{A.TIME SERIES ANALYSIS}

A Time series (TS) is a sequence of observations in ordered time. Mostly these observations are collected at equally spaced discrete time intervals. The basic assumption in any time series analysis modeling is that some aspects of the past pattern will continue to remain in the future. Also under this setup often the time series process is assumed to be based on past values of the main variable but not on explanatory variables which may affect the system. So the system act as a black box and we may only be able to know 'what' will happen rather than 'why' it happens. Here it is deliberately assumed that information about the past is available in the form of numerical data. Ideally at least 50 observations are necessary for performing time series analysis/modeling as propounded by Box and Jenkins who were pioneers in TS modeling [9].

Time series analysis can be used more easily for weather forecasting purposes because historical sequences of observations are readily available from published sources. All these successive observations are statistically dependent and Time series modeling is concerned with the techniques for analysis of such dependencies [9].

\section{B.MATLAB}

The name MATLAB stands for matrix laboratory. MATLAB is a high-performance language for technical computing. It integrates computation, visualization, and programming in an easy-to-use environment where problems and solutions are expressed in familiar mathematical notation. MATLAB is an interactive system whose basic data element is an array that does not require 
dimensioning. This allows you to solve many technical computing problems, especially those with matrix and vector formulations, in a fraction of the time.

\section{DATA COLLECTION}

Weather data of hundred years for the meteorological variables as shown in Table 1 were collected from the Indian Meteorological Department (IMD)[10,11]. The ellicitated data refers to two sets i.e. average maximum temperature and average minimum temperature for 12 months of each year. The general Structure of inputs/outputs for the network is shown in Fig3. The global data is divided into two groups, the training group corresponding to $60 \%$ of the data and the test group, corresponding to $40 \%$ of the data; so that the generalization capacity of the network could be verified after the training phase.

Mean Squared Error (MSE) is used as a measure of error made by the proposed network.

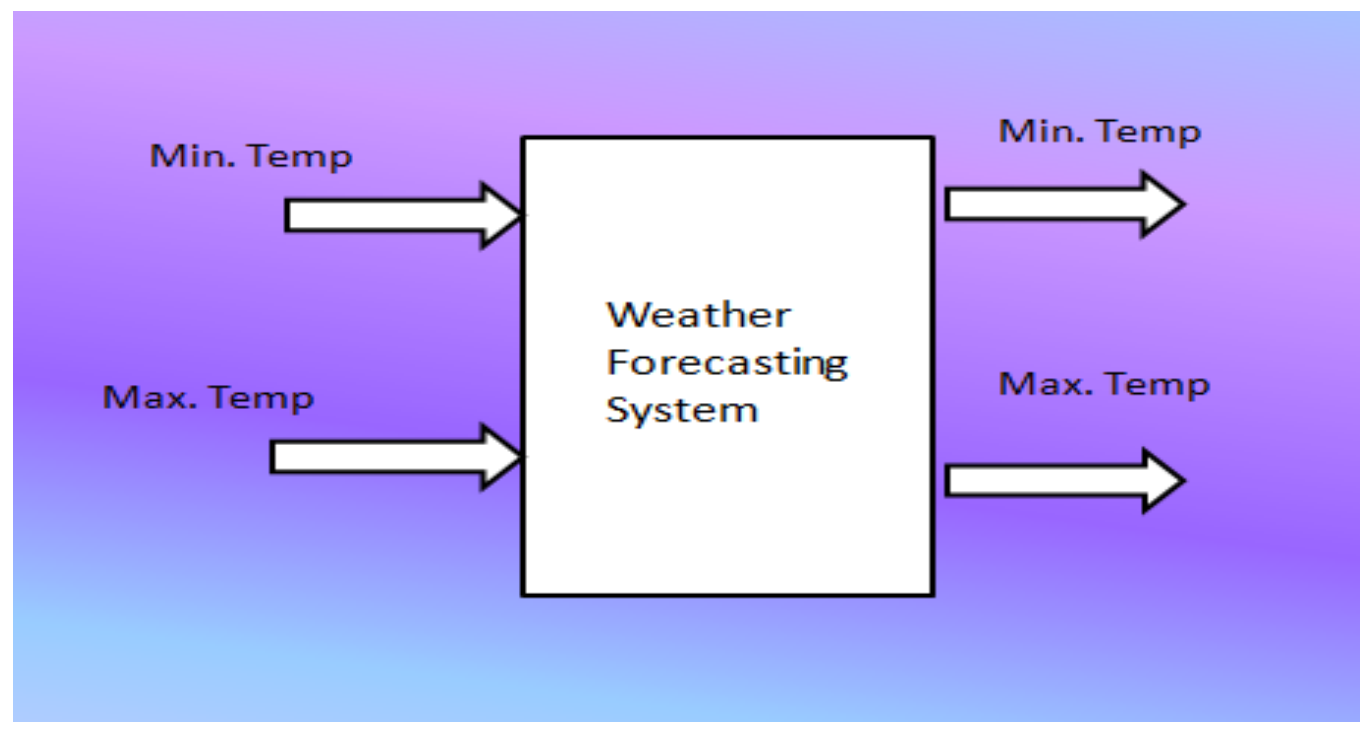

Fig 3: General Structure of inputs/outputs for the network.

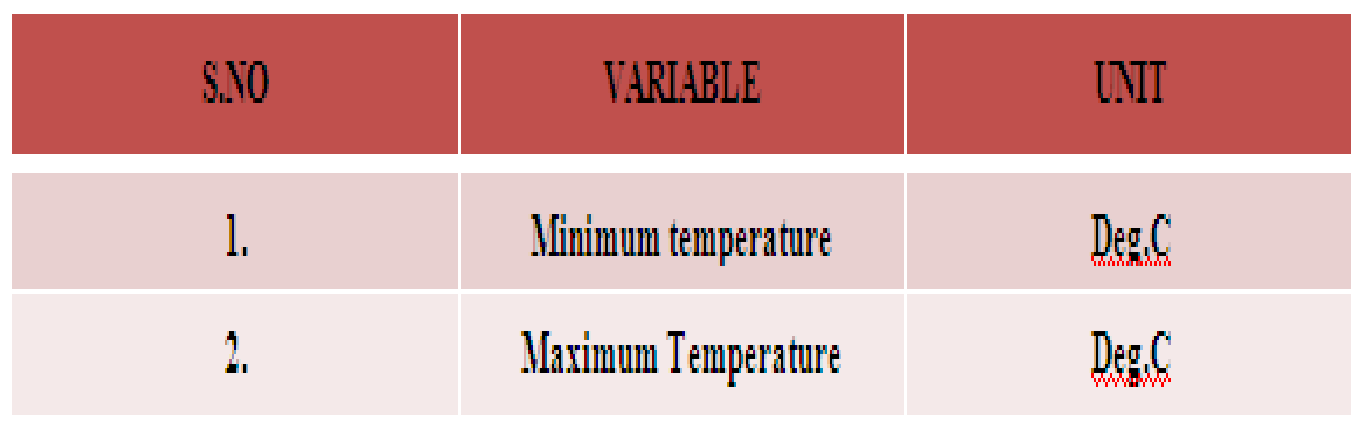

Table 1: Meteorological Variables. 


\section{RESULTS AND DISCUSSION}

\begin{tabular}{|l|l|}
\hline \multicolumn{2}{|c|}{ Network Structure } \\
\hline Number of Hidden neurons & 3 \\
\hline Number of epochs & 100 \\
\hline Activation function usedin hidden layer & tan-sig \\
\hline Activation function used in output layer & Pure linear \\
\hline
\end{tabular}

The obtained optimal network structure is shown in Table 2 below:

Table 2: The optimal Network Structure.

The network is trained with 60 years of data for both maximum and minimum temperature. After training phase the network is simulated by using $40 \%$ of data so that the predicted result can be verified.

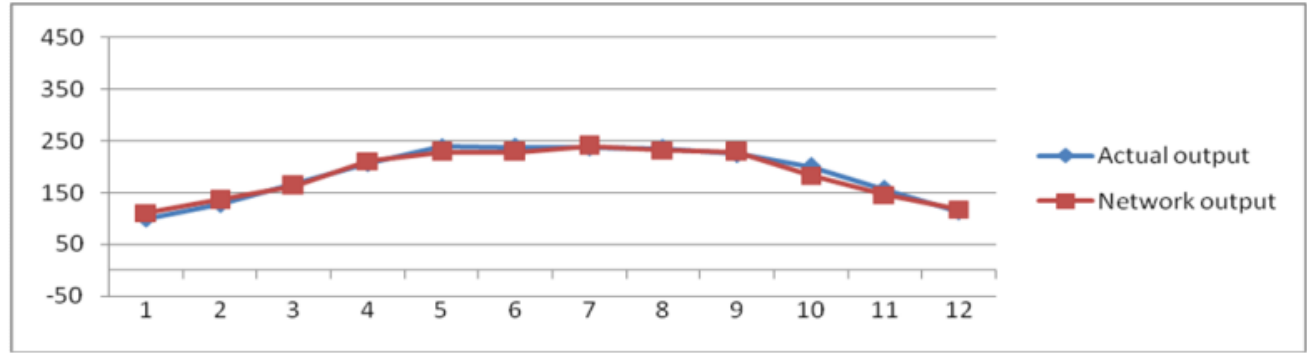

Fig 4: Comparison between actual and predicted minimum temperature values for year 2001 .

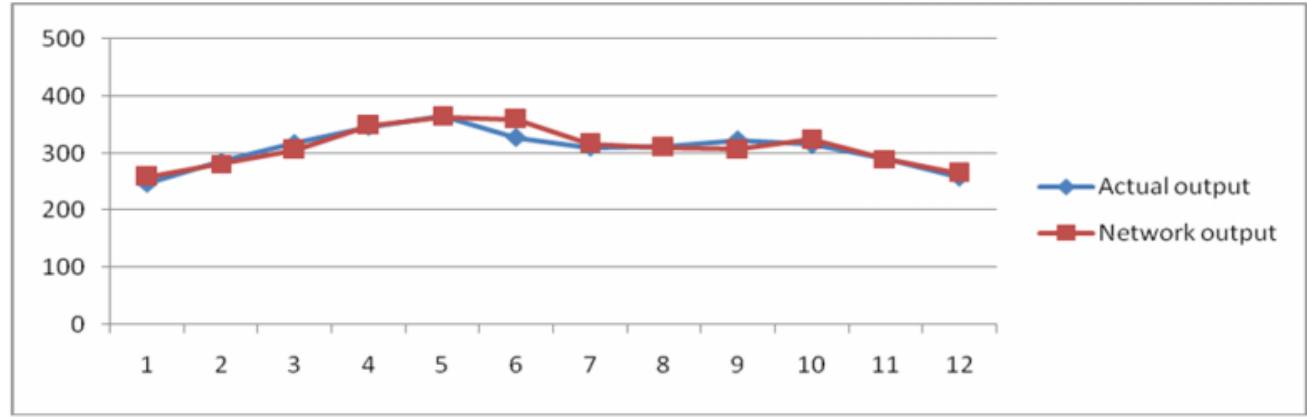

Fig 5: Comparison between actual and predicted maximum temperature values for year 2001

\section{CONCLUSION}

The results conclude that Multilayered Neural Network can be an effective tool in weather prediction. This type of Network can correctly provides the mapping between input and the output using historical data. Once the number of layers and units in each layer has been selected the network's weights and thresholds must be set so as to minimise the prediction error made by the network. The forecasting reliability was evaluated by comparing the actual and predicted 
temperature values. The results show that the network may be an important tool for temperature forecasting also.

\section{REFERENCES:}

1. Paras, S.Mathur, A.Kumar and M.Chandra (2007), "A Feature Based Neural Network Model for Weather Forecasting",World Academy of Science, Engineering and Technology.

2. Mr.R.C vashishtha, director IMD.

3. S N Sivanandam, S Sumathi, S N Deepa, "Introduction to neural Networks using MATLAB".

4. http://en.wikipedia.org/wiki/Neural_network.

5. Rosenblatt, Frank. x.(1961),"Principles of Neurodynamics: Perceptrons and the Theory of Brain Mechanisms", Spartan Books, Washington DC.

6. Rumelhart, David E., Geoffrey E. Hinton, and R. J. Williams (1986),"Learning Internal Representations by Error Propagation". David E. Rumelhart, James L. McClelland, and the PDP research group. (editors), Parallel distributed processing: Explorations in the microstructure of cognition, Volume 1: Foundations. MIT Press.

7. Cybenko, G. (1989), "Approximation by superpositions of a sigmoidal function Mathematics of Control, Signals, and Systems (MCSS)", 2(4), 303-314.

8. http://en.wikipedia.org/wiki/Multilayer_perceptron.

9. Ramasubramanian V."Time Series Analysis", I.A.S.R.I,Library Avenue,New Delhi.

10. http://www.tropmet.res.in.

11. http://www.imd.gov.in.

12. Chattopadhyay S. (2006), "Multilayered feed forward Artificial Neural Network model to predict the average summer-monsoon rainfall in India",IEEE ,Vol.:11, pp.:125-130

13. Zan C. (2009) "Myanmar Rainfall Forecasting Using Hidden Markov Model",IEEE International Advance Computing Conference.

14. Hayati M. and Mohebi Z.(2007), "Temperature Forecasting Based on neural Network Approach", World Applied Sciences Journal 2 (6):613-620.ISSN 1818-4952. 